\title{
Magnetic nanoscale laminates with tunable exchange coupling from first principles
}

\author{
Martin Dahlqvist, Björn Alling, Igor Abrikosov and Johanna Rosén
}

\section{Linköping University Post Print}

N.B.: When citing this work, cite the original article.

Original Publication:

Martin Dahlqvist, Björn Alling, Igor Abrikosov and Johanna Rosén, Magnetic nanoscale laminates with tunable exchange coupling from first principles, 2011, Physical Review B. Condensed Matter and Materials Physics, (84), 22, 220403.

http://dx.doi.org/10.1103/PhysRevB.84.220403

Copyright: American Physical Society http://www.aps.org/

Postprint available at: Linköping University Electronic Press http://urn.kb.se/resolve?urn=urn:nbn:se:liu:diva-73313 


\title{
Magnetic nanoscale laminates with tunable exchange coupling from first principles
}

\author{
M. Dahlqvist, ${ }^{*}$ B. Alling, I. A. Abrikosov, and J. Rosen \\ Department of Physics, Chemistry, and Biology (IFM), Linköping University, SE-581 83 Linköping, Sweden
}

(Received 28 June 2011; published 5 December 2011)

\begin{abstract}
The $M_{n+1} A X_{n}(M A X)$ phases are nanolaminated compounds with a unique combination of metallic and ceramic properties, not yet including magnetism. We carry out a systematic theoretical study of potential magnetic $M A X$ phases and predict the existence of stable magnetic $\left(\mathrm{Cr}_{1-x} \mathrm{Mn}_{x}\right)_{2} \mathrm{AlC}$ alloys. We show that in this system ferromagnetically ordered $\mathrm{Mn}$ layers are exchange coupled via nearly nonmagnetic Cr layers, forming an inherent structure of atomic-thin magnetic multilayers, and that the degree of disorder between $\mathrm{Cr}$ and $\mathrm{Mn}$ in the alloy can be used to tune the sign and magnitude of the coupling.
\end{abstract}

DOI: 10.1103/PhysRevB.84.220403

PACS number(s): 75.70.Cn, 71.15.Mb, 75.50.Gg

Layered magnetic materials are attractive from the point of view of fundamental science and applications. Interest in their study was boosted by the observation of interlayer exchange coupling in magnetic multilayers, ${ }^{1}$ followed by the discoveries of the giant magnetoresistance $(\mathrm{GMR})^{2,3}$ effect, which revolutionized applications such as data storage and magnetic recording, and have launched the vast field of spintronics. ${ }^{4}$ Extensive research on multilayers composed of magnetic and nonmagnetic material includes, for example, magnetoresistance ratios, ${ }^{5,6}$ magnetic switching, ${ }^{7}$ thermal stability, ${ }^{8,9}$ and scalability. ${ }^{8}$ The resulting layered magnetic structures in various applications are, in general, on the nanosize level. Engineering will show what currently used materials can achieve, and when, and at what feature size other materials are needed. Here we identify, to the best of our knowledge, a previously unknown class of atomic-thin magnetic layered materials based on $M_{n+1} A X_{n}(M A X)$ phase nanolaminated ternary compounds, using first-principles calculations to study trends in phase stability for potential magnetic $M A X$ phases. A stable magnetic phase, the $\left(\mathrm{Cr}_{1-x} \mathrm{Mn}_{x}\right)_{2} \mathrm{AlC}$ alloy, is identified, and its magnetic and electronic properties are evaluated. We observe that ferromagnetically ordered $\mathrm{Mn}$ layers are exchange coupled via nearly nonmagnetic $\mathrm{Cr}$ layers, forming an inherent structure of atomic-thin magnetic multilayers. We also show that the exchange coupling in this system can be tuned via a control of degree of intermixing between $\mathrm{Mn}$ and $\mathrm{Cr}$ in their respective layers. The strongly anisotropic electronic structure, combined with a tunable exchange coupling and thermodynamic stability, give these materials high potential for electronics and spintronics applications.

$M A X$ phases represent a class of naturally nanolaminated materials with a common formula $M_{n+1} A X_{n}(n=1-3)$, where $M$ is an early transition metal, $A$ is an $A$-group element, and $X$ is carbon or nitrogen. ${ }^{10}$ The hexagonal structure, which belongs to the $P 6_{3} / m m c$ (No. 194) space group, can be described as $M_{n+1} X_{n}$ layers interleaved with an atomic layer of $A$ elements, which for $n=1$ results in a $M-X-M-A-M-X-M-$ $A$ atomic-layer stacking in the $c$ direction. These compounds have lately attracted extensive attention due to their unique combination of physical, chemical, electrical, and mechanical properties, combining the characteristics of ceramics and metals. ${ }^{11}$ In addition, vastly anisotropic optical and electronic properties are predicted, with tunable conductivity in the $c$ direction. ${ }^{12}$ Combining the inherent nanolaminated structure and known characteristics, such as high stability, high wear resistance, and highly anisotropic properties, with magnetism, would potentially give rise to functional materials for various spintronics applications. To date, there are more than 60 known $M_{n+1} A X_{n}$ phases. ${ }^{13}$ However, to the best of our knowledge, none have experimentally been demonstrated to show magnetic behavior. The few theoretical studies available ${ }^{14-16}$ have not been able to identify suitable thermodynamically stable candidates, and hence the task of predicting a stable magnetic $M A X$ phase is still open.

Throughout this work, a $b$ initio calculations based on density functional theory (DFT) were performed using the projector augmented-wave (PAW) method ${ }^{17}$ as implemented within VASP. ${ }^{18,19}$ The Perdew-Burke-Ernzerhof $(\mathrm{PBE})^{20}$ generalized gradient approximation (GGA) was used for the exchange and correlation functional in its spin-polarized version. Reciprocal-space integration was performed within the Monkhorst-Pack scheme ${ }^{21}$ with a plane-wave cutoff energy of $400 \mathrm{eV}$. The calculated total energy for all phases included in the present Rapid Communication are converged to within $0.1 \mathrm{meV} /$ atom in terms of $k$-point sampling and plane-wave energy cutoff. Structural optimizations were performed in terms of unit-cell volumes, $c / a$ ratios (when necessary), and internal parameters to minimize the total energy. Nonmagnetic (NM), ferromagnetic (FM), and different antiferromagnetic (AFM) states were tested for each phase and the configuration with lowest energy was included in the study.

Based on a recently developed procedure, ${ }^{22,23}$ here the stability of potential magnetic $M_{n+1} \mathrm{AlC}_{n}$ phases was systematically evaluated for $M=\mathrm{Cr}, \mathrm{Mn}, \mathrm{Fe}$, and $\mathrm{Co}$ in NM, FM, and different AFM states. In order to include known as well as hypothetical competing phases, careful investigations of phase diagrams (see, e.g., Ref. 24), including both experimental and theoretical work, were carried out. A linear optimization procedure ${ }^{22,23}$ was then used to identify the set of most competing phases with respect to each $M_{n+1} \mathrm{AlC}_{n}$ phase. The calculated energy differences in the present study are converged to within $0.1 \mathrm{meV} /$ atom. Expressed in total energy per atom (in $\mathrm{eV}$ ), the formation enthalpy $\Delta H_{\mathrm{cp}}$ with respect to the identified most competing phases (cp) at zero pressure and temperature is calculated according to $\Delta H_{\mathrm{cp}}=$ $H\left[M_{n+1} \mathrm{AlC}_{n}\right]-H_{\text {total }}[\mathrm{cp}]$. 


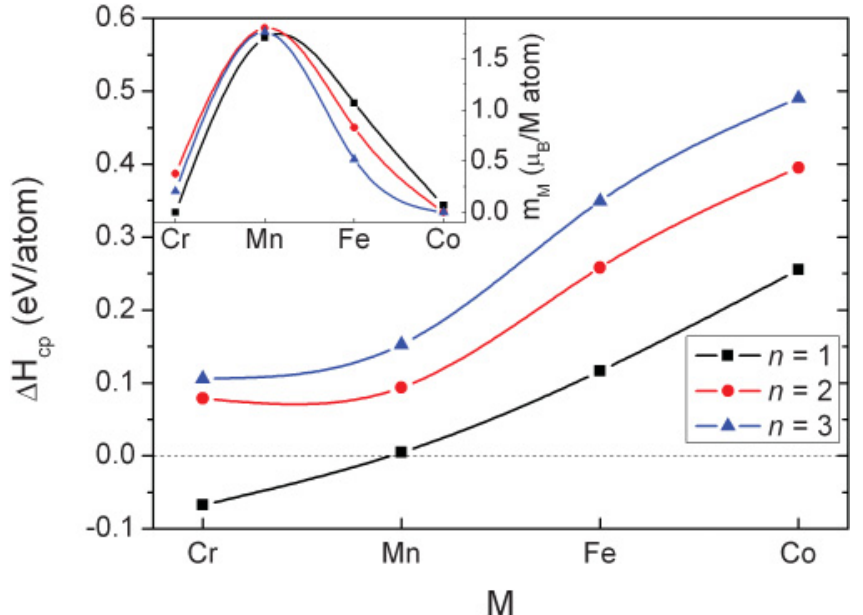

FIG. 1. (Color online) Formation enthalpy $\Delta H_{\mathrm{cp}}$ of $M_{n+1} \mathrm{AlC}_{n}$ for $M=\mathrm{Cr}, \mathrm{Mn}, \mathrm{Fe}$, and $\mathrm{Co}$, and $n=1-3$. Negative values indicate thermodynamic stability of the corresponding $M A X$ phase with respect to decomposition into most competitive phases (cp). The inset shows the $M$-site local magnetic moment $\mu_{M}$ in units of Bohr magneton $\mu_{B}$.

The results for $M=\mathrm{Cr}, \mathrm{Mn}, \mathrm{Fe}$, and $\mathrm{Co}$ systems are presented in Fig. 1 for $M_{n+1} \mathrm{AlC}_{n}$ phases in their lowestenergy magnetic states. $\Delta H_{\mathrm{cp}}$ increases as the valence of the $M$ element increases, for all $n . \mathrm{Cr}_{2} \mathrm{AlC}$ is identified as the only stable $M_{n+1} \mathrm{AlC}_{n}$ phase, in accordance with experimental observations, with a negative $\Delta H_{\mathrm{cp}}$ of $-0.067 \mathrm{eV} /$ atom. However, $\mathrm{Mn}_{2} \mathrm{AlC}$ is very close to thermodynamic stability with $\Delta H_{\mathrm{cp}}$ being just $+0.005 \mathrm{eV} /$ atom. Here, the most competing phases, out of all the phases included, ${ }^{23}$ are identified as $\mathrm{Mn}_{3} \mathrm{AlC}, \mathrm{MnAl}$, and C. $\mathrm{Fe}_{2} \mathrm{AlC}$ is found to be quite metastable with $\Delta H_{\mathrm{cp}}=+0.116$ $\mathrm{eV} /$ atom, and it should decompose into the inverse perovskite, $\mathrm{Fe}_{3} \mathrm{AlC},{ }^{25,26}$ in combination with $\mathrm{FeAl}$ and $\mathrm{C}$. The discrepancy with previously reported results on this system ${ }^{14}$ highlights the need to include all competing phases in phase stability studies. The trend in increasing instability is continued with $\mathrm{Co}_{2} \mathrm{AlC}$, where the most competitive phases are $\mathrm{CoAl}, \mathrm{Co}$, and $\mathrm{C}$. As $\Delta H_{\mathrm{cp}}$ is comparatively large for all phases with $n=2$ and 3 , they will not be further discussed in this work.

In the inset of Fig. 1 the magnetic moment $\mu_{B}$ per $M$ element is presented. Unfortunately, the stable $\mathrm{Cr}_{2} \mathrm{AlC} M A X$ phase is found to be NM, in agreement with experiments. The magnetic moment is, however, maximized at the Mn-based $M_{2} \mathrm{AlC}$ phase. Based on this observation, and on the high stability of the well-known $\mathrm{Cr}_{2} \mathrm{AlC}$, we suggest alloying as a strategy to obtain a stable FM MAX phase. Hence, our efforts have been concentrated to a mixture of $\mathrm{Mn}$ and $\mathrm{Cr}$ on the $M$ sublattice, represented as the alloy $\left(\mathrm{Cr}_{1-x} \mathrm{Mn}_{x}\right)_{2} \mathrm{AlC}$, with $x=0.25,0.50$, and 0.75 . The identified set of most competitive phases at all $x$ is $\mathrm{Cr}_{2} \mathrm{AlC}, \mathrm{Mn}_{3} \mathrm{AlC}, \mathrm{MnAl}$, and $\mathrm{C}$. The hypothetical $\mathrm{Cr}_{3} \mathrm{AlC}$, as well as the mixed $\left(\mathrm{Cr}_{0.5} \mathrm{Mn}_{0.5}\right)_{3} \mathrm{AlC}$ inverse perovskite, in NM, FM, and different AFM states, have also been considered but are energetically unfavorable. Several ordered as well as disordered (with respect to $\mathrm{Mn}$ and $\mathrm{Cr}$ intermixing) atomic configurations have been studied in different magnetic states, including NM, FM, various AFM, double-layer antiferromagnetic (dAFM), and paramagnetic (PM). The latter state was approximated by means of the disorder local moment (DLM) ${ }^{27,28}$ model, where the $\operatorname{DLM}\left(\mathrm{Cr}_{0.5} \mathrm{Mn}_{0.5}\right)_{2} \mathrm{AlC}$ phase was represented by a random configuration of $\mathrm{Mn}$ atoms with up $\mathrm{Mn}^{\uparrow}$ and down $\mathrm{Mn}^{\downarrow}$ spins in a $\left(\mathrm{Cr}_{0.5} \mathrm{Mn}_{0.25}{ }^{\uparrow} \mathrm{Mn}_{0.25}{ }^{\downarrow}\right)_{2} \mathrm{AlC}$ alloy.

For the disordered $M$ element configurations, an appropriate supercell was chosen based on the criterion to mimic an atomic distribution in a random alloy, i.e., $M$-site correlation functions equal to zero. This is achieved by simulating a solid solution of $\mathrm{Cr}$ and $\mathrm{Mn}$ on the $M$ sublattice by the so-called special quasirandom structures (SQS) method, as suggested by Zunger et al. $^{29}$ and introduced for $M A X$ phases by us. ${ }^{22}$ Supercells ranging from a $1 \times 1 \times 1$ unit cell (four $M$ sites) to $4 \times 2 \times 1$ or $2 \times 2 \times 2$ unit cells ( $32 M$ sites) were generated for $\left(\mathrm{Cr}_{1-x} \mathrm{Mn}_{x}\right)_{2} \mathrm{AlC}$ and evaluated for all $x$. With a convergence energy of less than $0.002 \mathrm{eV} /$ atom for the $4 \times 2 \times 1 \mathrm{SQS}$ supercells, these were used throughout the work to represent the disordered $M$ configurations. It should be noted that though several ordered atomic configurations have been considered for all $x$, those with pure Mn-C-Mn layers have been found to have the lowest energy. Accordingly, the most energetically stable ordered structure for $x=0.50$, among all those studied, consists of a layered distribution of $\mathrm{Mn}$ and $\mathrm{Cr}$, resulting in $\mathrm{Mn}-\mathrm{C}-\mathrm{Mn}$-Al-Cr-C-Cr-Al stacking in the $c$ direction, hereafter referred to as $\mathrm{Mn}-\mathrm{C}-\mathrm{Mn}$.

In Fig. 2(a) the formation enthalpy $\Delta H_{\mathrm{cp}}$ of $\mathrm{FM}$ $\left(\mathrm{Cr}_{1-x} \mathrm{Mn}_{x}\right)_{2} \mathrm{AlC}$ is shown for the identified most stable ordered configurations (squares) and for the configurationally

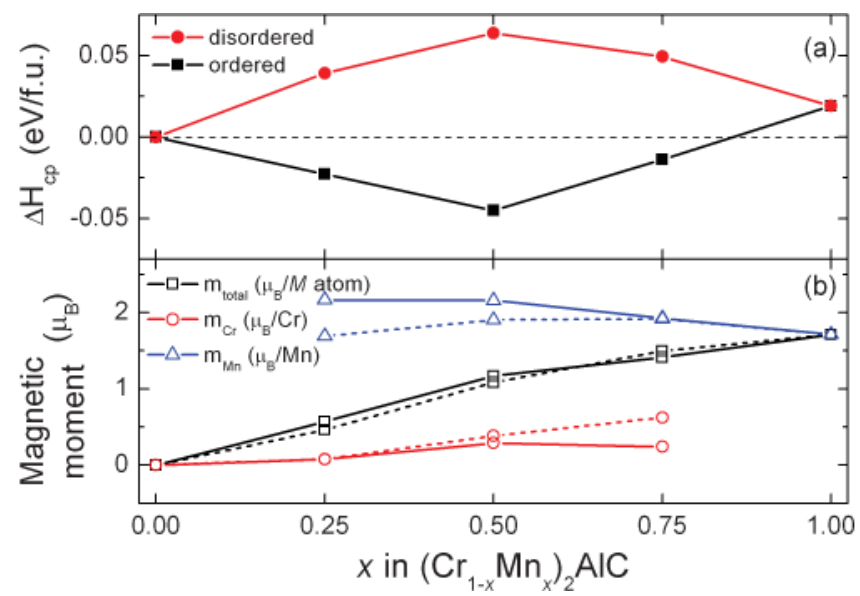

FIG. 2. (Color online) (a) Formation enthalpy $\Delta H_{\mathrm{cp}}$ of FM $\left(\mathrm{Cr}_{1-x} \mathrm{Mn}_{x}\right)_{2} \mathrm{AlC}$. Results are presented for chemically disordered (circles) and the identified most stable ordered structures (squares) at all $x$. (b) Average magnetic moment per $M$ atom in $\mu_{B}$ for $\left(\mathrm{Cr}_{1-x} \mathrm{Mn}_{x}\right)_{2} \mathrm{AlC}$ (squares), and average local moments at $\mathrm{Cr}$ (circles) and $\mathrm{Mn}$ atoms (triangles). The identified most stable chemically ordered structures are represented by solid lines, while solid solutions are represented by dashed lines. An increase in the net moment per $M$ atom with increasing $x$ is evident for ordered as well as disordered systems. The difference between ordered and random atomic configurations are most pronounced for $\mathrm{Cr}$ at $x=0.75$, with the $\mathrm{Cr}$ moments induced by the surrounding $\mathrm{Mn}$ atoms in the solid solution, and for $\mathrm{Mn}$ at $x=0.25$, where $\mathrm{Mn}-\mathrm{Mn}$ nearest neighbors are more frequent in the ordered structure. 
disordered systems (circles) as a function of $x$. Note that $\Delta H_{\mathrm{cp}}$ is negative for the ordered systems only, with a minimum of $-0.045 \mathrm{eV} /$ f.u. at $x=0.50$ for the Mn-C-Mn configuration. The corresponding lattice parameters $a$ and $c$ have increased by $\sim 0.5 \%$ and decreased by $\sim 0.7 \%$, respectively, as compared to relaxed $\mathrm{Cr}_{2} \mathrm{AlC}$. Moreover, the $c / a$ ratio decreases almost linearly from $4.47(x=0.00)$ to $4.36(x=1.00)$. For the disordered $M$ alloys, positive values of $\Delta H_{\mathrm{cp}}$ are found for all $x$, with a maximum of $+0.064 \mathrm{eV} /$ f.u. at $x=0.50$. However, at finite temperature, the configurational entropy due to the disorder (with respect to $M$ elements) in the solid solution makes it more compatible with respect to chemically ordered states, as well as with respect to decomposition into competing ordered phases. It is therefore justified to consider magnetic properties of ordered as well as disordered phases in the continued discussion.

The average magnetic moment per $M$ atom of ferromagnetic $\left(\mathrm{Cr}_{1-x} \mathrm{Mn}_{x}\right)_{2} \mathrm{AlC}$ is shown in Fig. 2(b), together with the average local moments at $\mathrm{Cr}$ and $\mathrm{Mn}$ atoms. The figure clearly shows that the major contribution to magnetism in this system comes from the $\mathrm{Mn}$ atoms, while $\mathrm{Cr}$ is nearly nonmagnetic in the chemically ordered (layered) structures. The $\mathrm{Cr}$ moments are smaller and less stable than the $\mathrm{Mn}$ moments, an effect which is most pronounced for disordered systems at $x=0.25$, where their individual spread is largest. The presented observations make the investigated material resemble magnetic multilayers, though with the interlayer spacing decreased from the nanolevel to the atomic scale. To complete this analogy, we must answer several questions: What is the magnetic ground state of $\left(\mathrm{Cr}_{1-x} \mathrm{Mn}_{x}\right)_{2} \mathrm{AlC}$ ? How stable is it with respect to magnetic disorder? Is there an exchange coupling between the atomic-thin magnetic layers, and can it be controlled? We consider these questions below.

A detailed evaluation of the energies of different magnetic states is shown in Fig. 3 for chemically ordered and increasingly disordered (with respect to the $M$ element) configurations in the $\left(\mathrm{Cr}_{0.5} \mathrm{Mn}_{0.5}\right)_{2} \mathrm{AlC}$ alloy. For the ordered $\mathrm{Mn}-\mathrm{C}-\mathrm{Mn}$ case we find that the magnetic ground state is degenerate between FM and AAFM. The latter consists of ferromagnetically ordered double layers of $\mathrm{Mn}$ moments in each Mn-C-Mn atomic trilayer, while the order between the double layers, separated by two $\mathrm{Al}$ atomic layers and one $\mathrm{Cr}-\mathrm{C}-\mathrm{Cr}$ trilayer, is antiparallel [Fig. 3(c), right-hand side]. The energy difference between these states is very small, with the dAFM order being $\sim 0.002 \mathrm{eV} /$ f.u. lower than the FM one. Note that they are both considerably lower in energy, 0.094 and $0.092 \mathrm{eV} /$ f.u., respectively, than the PM state, approximated by the DLM solution. In the $M$-site solid solution, on the other hand, the FM order is clearly more stable than both the dAFM (0.017 eV/f.u.) and the different AFM orders. Furthermore, the energy difference between the FM and DLM state in this case is $0.040 \mathrm{eV} / \mathrm{f}$.u. It should be noted that the supercells made to describe the dAFM and DLM magnetic order, respectively, could not be constructed with an identical atomic configuration. Despite this, the calculated magnetic energy differences can be directly compared since the energies of the FM state for the two atomic configurations differ only by $0.004 \mathrm{eV} /$ f.u. Nevertheless, the difficulties of theoretically resolving exact magnetic ground states for disordered systems, dictated by a delicate balance of varying magnetic interactions

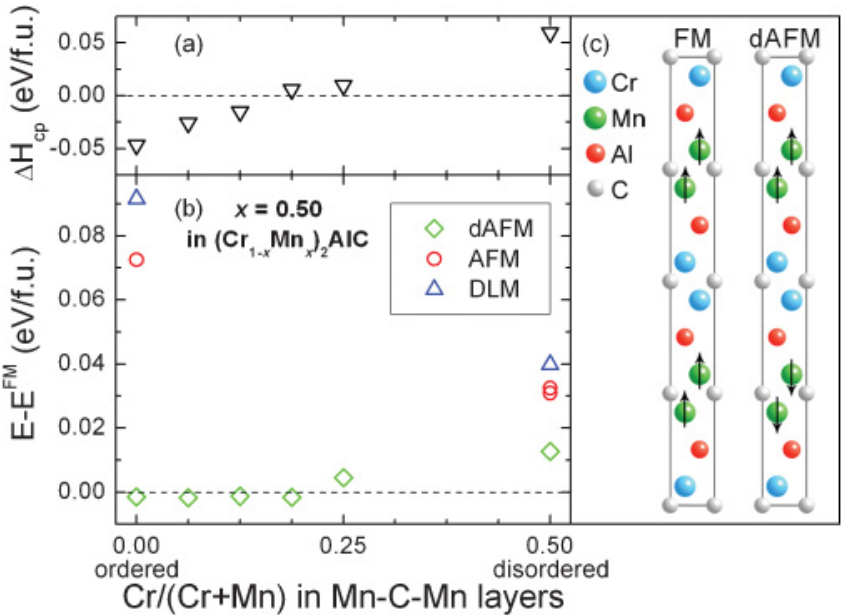

FIG. 3. (Color online) (a) Formation enthalpy $\Delta H_{\mathrm{cp}}$ of $\left(\mathrm{Cr}_{0.5} \mathrm{Mn}_{0.5}\right)_{2} \mathrm{AlC}$ in its identified most stable magnetic states, and (b) the energy of dAFM, AFM, and PM states relative to the FM state, as a function of the degree of $M$-site intermixing, measured as $\mathrm{Cr}$ concentration in $\mathrm{Mn}-\mathrm{C}-\mathrm{Mn}$ layers. The intermixing ranges from 0 , for the identified most stable ordered structure with Mn-C-Mn and $\mathrm{Cr}-\mathrm{C}-\mathrm{Cr}$ layers separated by an $\mathrm{Al}$ layer as shown in (c) for the FM (left) and dAFM (right) magnetic states, to full disorder at 0.5, represented by a special quasirandom structure (SQS) supercell (see Ref. 20). The first and last points in (a) correspond to the values of $\Delta H_{\mathrm{cp}}$ given in Fig. 2(a) for $x=0.50$.

as well as chemical local environments, should be kept in mind.

From the energy differences between ordered and completely disordered magnetism, the magnetic ordering temperature could be qualitatively estimated. For instance, the cubic $\mathrm{Ti}_{0.5} \mathrm{Cr}_{0.5} \mathrm{~N}$ solid solution with an experimental Curie temperature between 140 and $170 \mathrm{~K},{ }^{30,31}$ was recently calculated to have an energy difference between the FM and DLM states of $0.041 \mathrm{eV}$ per magnetic atom. ${ }^{32}$ This is close to the result presented here for the chemically disordered state [see Fig. 3(b)]. The much larger energy gain by magnetic ordering in the Mn-C-Mn ordered state (a factor of 2.3 larger) indicates that partially ordered $\left(\mathrm{Cr}_{0.5} \mathrm{Mn}_{0.5}\right)_{2} \mathrm{AlC}$, not to mention compositions somewhat richer in Mn, may be FM also above room temperature.

An important observation should be made at this point: There is an exchange coupling between ferromagnetic-inthe-layer ordered Mn-rich layers in the $\left(\mathrm{Cr}_{0.5} \mathrm{Mn}_{0.5}\right)_{2} \mathrm{AlC}$ alloy, which can be either FM or AFM, depending on the degree of chemical disorder in the system. This observation strengthens an analogy between our system and exchangecoupled magnetic multilayers. ${ }^{1}$ In the latter case, the exchange coupling can be tuned by the thickness of the spacer layer. We suggest another tuning mechanism for our system. Indeed, the consequences of a gradual chemical disordering of the ordered Mn-C-Mn configuration, which could be affected via, for instance, control of synthesis conditions, are shown in Fig. 3(b). With increasing intermixing between $\mathrm{Cr}$ and $\mathrm{Mn}$ atoms, the FM order becomes more favorable than the dAFM-based magnetic order. These results suggest a path for tailoring the exchange coupling between the Mn-rich layers, where the degree of disorder is used as a tuning parameter, allowing systems 


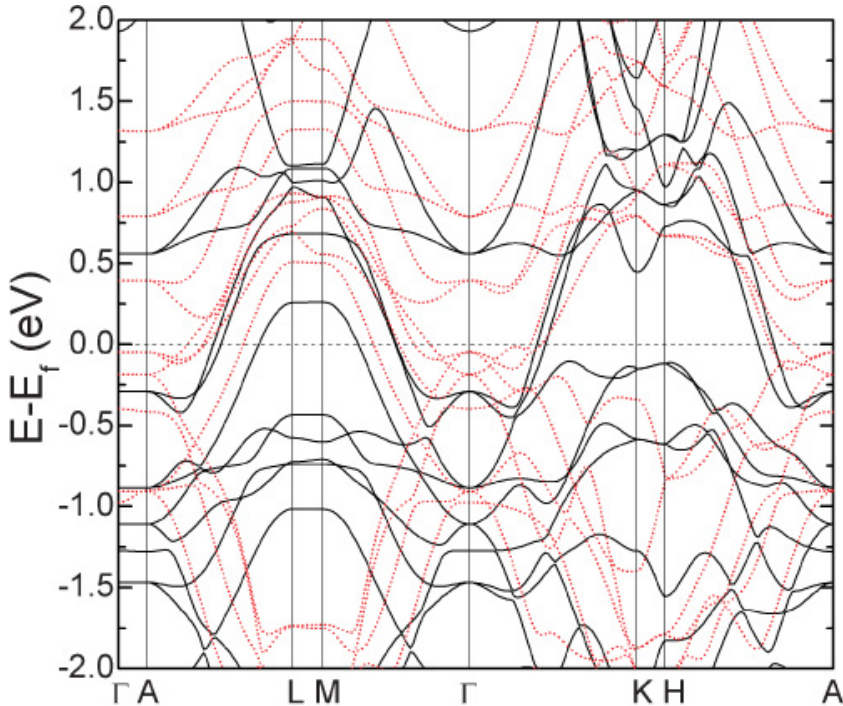

FIG. 4. (Color online) The electronic band structure of FM $\left(\mathrm{Cr}_{0.5} \mathrm{Mn}_{0.5}\right)_{2} \mathrm{AlC}$ (Mn-C-Mn structure) for high-symmetry points with spin-up and spin-down states represented by solid black lines and dotted red lines, respectively.

ranging from a dAFM magnetic configuration [Fig. 3(c), right-hand side] to a FM configuration [Fig. 3(c), left-hand side] through a situation with a completely degenerate FM and dAFM configuration at $\sim 20 \% \mathrm{Cr}$ admixture. A correlated tuned response to small external magnetic fields is expected, which is highly valuable for materials used in spintronics applications.

An electronic structure analysis for the FM Mn-C-Mn configuration is presented in Fig. 4. The band structure shows metallic behavior in the basal plane, with numerous bands crossing the Fermi level. In the $c$ direction, corresponding to the $\Gamma-A, \mathrm{~L}-\mathrm{M}$, and $\mathrm{K}-\mathrm{H}$ directions in reciprocal space, no bands cross the Fermi level, and a dispersionless flatband character is evident. Hence, vastly anisotropic electrical properties are indicated. The same band characteristics, especially in the $\Gamma-A$ direction, are found also for the ordered dAFM configuration.

To date, and to the best of our knowledge, there are no thermodynamically stable magnetic $M A X$ phase materials known. However, the results presented here predict a magnetic nanolaminate, $\left(\mathrm{Cr}_{1-x} \mathrm{Mn}_{x}\right)_{2} \mathrm{AlC}$, as a stable magnetic $M A X$ phase with tunable exchange coupling between ferromagnetically ordered Mn-rich layers. In contrast to the conventional approach for the control of exchange coupling by varying the interlayer thickness ${ }^{1}$ and the interface quality, ${ }^{5}$ our results suggest that one can vary the $M$-element alloy composition as well as the degree of $M$-element intermixing to control the interlayer coupling. Taking advantage of the naturally layered structure of these materials and their highly anisotropic transport properties, which can additionally be tuned by $X$-element alloying, ${ }^{12}$ there is a potential to use magnetic $M A X$ phases for electronic and spintronic applications. Separately, or stacked together, the properties within each $M A X$ phase layer can be tailored to optimize the performance. This concept can be realized in material synthesis with atomic-layer control, combining no interface mismatch and improved scalability with tuned magnetism, the latter by means of adjusting growth parameters, such as temperature, and hence promote structures and properties governed by self-organization.

The research leading to these results have received funding from the European Research Council under the European Community's Seventh Framework Programme (FP7/20072013)/ERC Grant agreement No. [258509], from the Centre in Nanoscience and Technology (CeNano), and from the Swedish Research Council (VR). I.A.A. acknowledges funding from the Swedish e-Science Research Centre (SeRC), the research center "Advanced Functional Materials (AFM)," and the Göran Gustafsson Foundation for Research in Natural Sciences and Medicine. The calculations were performed using computational resources allocated by the Swedish National Infrastructure for Computing (SNIC). *madah@ifm.liu.se

${ }^{1}$ P. Grünberg, R. Schreiber, Y. Pang, M. B. Brodsky, and H. Sowers, Phys. Rev. Lett. 57, 2442 (1986).

${ }^{2}$ M. N. Baibich, J. M. Broto, A. Fert, F. Nguyen Van Dau, F. Petroff, P. Etienne, G. Creuzet, A. Friederich, and J. Chazelas, Phys. Rev. Lett. 61, 2472 (1988).

${ }^{3}$ G. Binasch, P. Grünberg, F. Saurenbach, and W. Zinn, Phys. Rev. B 39, 4828 (1989).

${ }^{4}$ S. A. Wolf, D. D. Awschalom, R. A. Buhrman, J. M. Daughton, S. von Molnar, M. L. Roukes, A. Y. Chtchelkanova, and D. M. Treger, Science 294, 1488 (2001).

${ }^{5}$ S. Ikeda, J. Hayakawa, Y. Ashizawa, Y. M. Lee, K. Miura, H. Hasegawa, M. Tsunoda, F. Matsukura, and H. Ohno, Appl. Phys. Lett. 93, 082508 (2008).

${ }^{6}$ S. S. P. Parkin, C. Kaiser, A. Panchula, P. M. Rice, B. Hughes, M. Samant, and S.-H. Yang, Nat. Mater. 3, 862 (2004).
${ }^{7}$ Y. Jiang, T. Nozaki, S. Abe, T. Ochiai, A. Hirohata, N. Tezuka, and K. Inomata, Nat. Mater. 3, 361 (2004).

${ }^{8}$ S. Ikeda et al., Nat. Mater. 9, 721 (2010).

${ }^{9}$ S. G. Lee and S. H. Lim, Appl. Phys. Lett. 97, 102501 (2010).

${ }^{10}$ M. W. Barsoum and T. El-Raghy, Am. Sci. 89, 334 (2001).

${ }^{11}$ M. W. Barsoum, Prog. Solid State Chem. 28, 201 (2000).

${ }^{12}$ J. Rosen, M. Dahlqvist, S. I. Simak, D. R. McKenzie, and M. M. M. Bilek, Appl. Phys. Lett. 97, 073103 (2010).

${ }^{13}$ P. Eklund, M. Beckers, U. Jansson, H. Högberg, and L. Hultman, Thin Solid Films 518, 1851 (2010).

${ }^{14}$ W. Luo and R. Ahuja, J. Phys. Condens. Matter 20, 064217 (2008).

${ }^{15}$ J. M. Schneider, Z. Sun, R. Mertens, F. Uestel, and R. Ahuja, Solid State Commun. 130, 445 (2004).

${ }^{16}$ W. Zhou, L. J. Liu, and P. Wu, J. Appl. Phys. 106, 7 (2009).

${ }^{17}$ P. E. Blöchl, Phys. Rev. B 50, 17953 (1994). 
${ }^{18}$ G. Kresse and J. Hafner, Phys. Rev. B 48, 13115 (1993).

${ }^{19}$ G. Kresse and J. Hafner, Phys. Rev. B 49, 14251 (1994).

${ }^{20}$ J. P. Perdew, K. Burke, and M. Ernzerhof, Phys. Rev. Lett. 77, 3865 (1996).

${ }^{21}$ H. J. Monkhorst and J. D. Pack, Phys. Rev. B 13, 5188 (1976).

${ }^{22}$ M. Dahlqvist, B. Alling, I. A. Abrikosov, and J. Rosén, Phys. Rev. B 81, 024111 (2010).

${ }^{23}$ M. Dahlqvist, B. Alling, and J. Rosén, Phys. Rev. B 81, 220102 (2010).

${ }^{24}$ H. Okamoto, Phase Diagrams for Binary Alloys (ASM International, Materials Park, OH, 2000).

${ }^{25}$ D. Connétable and P. Maugis, Intermetallics 16, 345 (2008).
${ }^{26}$ M. Palm and G. Inden, Intermetallics 3, 443 (1995).

${ }^{27}$ B. Alling, T. Marten, and I. A. Abrikosov, Phys. Rev. B 82, 184430 (2010).

${ }^{28}$ B. L. Gyorffy, A. J. Pindor, J. Staunton, G. M. Stocks, and H. Winter, J. Phys. F 15, 1337 (1985).

${ }^{29}$ A. Zunger, S. H. Wei, L. G. Ferreira, and J. E. Bernard, Phys. Rev. Lett. 65, 353 (1990).

${ }^{30}$ M. I. Aivazov and S. V. Gurov, Izv. Akad. Nauk SSSR, Neorg. Mater. 11, 1385 (1975).

${ }^{31}$ K. Inumaru, K. Koyama, Y. Miyaki, K. Tanaka, and S. Yamanaka, Appl. Phys. Lett. 91, 152501 (2007).

${ }^{32}$ B. Alling, Phys. Rev. B 82, 054408 (2010). 